\title{
A possible formation channel for blue hook stars in globular cluster - II. Effects of metallicity, mass ratio, tidal enhancement efficiency and helium abundance
}

\author{
Zhenxin Lei ${ }^{1,2 \star}$, Gang Zhao ${ }^{2} \dagger$, Aihua Zeng ${ }^{1}$, Lihua Shen $^{1}$, Zhongjian Lan ${ }^{1}$ \\ Dengkai Jiang 3,4 and Zhanwen $\operatorname{Han}^{3,4} \ddagger$ \\ ${ }^{1}$ Department of Science, Shaoyang University, Shaoyang 422000, China \\ ${ }^{2}$ Key Laboratory of Optical Astronomy, National Astronomical Observatories, Chinese Academy of Sciences, Beijing 100012, China \\ ${ }^{3}$ Key Laboratory for the Structure and Evolution of Celestial Objects, Chinese Academy of Sciences,Kunming 650011, China \\ ${ }^{4}$ Yunnan Observatory, Chinese Academy of Sciences, Kunming 650011, China
}

Accepted ; Received ; in original form

\begin{abstract}
Employing tidally enhanced stellar wind, we studied in binaries the effects of metallicity, mass ratio of primary to secondary, tidal enhancement efficiency and helium abundance on the formation of blue hook (BHk) stars in globular clusters (GCs). A total of 28 sets of binary models combined with different input parameters are studied. For each set of binary model, we presented a range of initial orbital periods that is needed to produce BHk stars in binaries. All the binary models could produce BHk stars within different range of initial orbital periods. We also compared our results with the observation in the $T_{\text {eff }}-\log g$ diagram of GC NGC 2808 and $\omega$ Cen. Most of the BHk stars in these two GCs locate well in the region predicted by our theoretical models, especially when $\mathrm{C} / \mathrm{N}$-enhanced model atmospheres are considered. We found that mass ratio of primary to secondary and tidal enhancement efficiency have little effects on the formation of BHk stars in binaries, while metallicity and helium abundance would play important roles, especially for helium abundance. Specifically, with helium abundance increasing in binary models, the space range of initial orbital periods needed to produce BHk stars becomes obviously wider, regardless of other input parameters adopted. Our results were discussed with recent observations and other theoretical models.
\end{abstract}

Key words: binaries: general - globular clusters: general

\section{INTRODUCTION}

Horizontal branch (HB) stars in globular clusters (GCs) are low mass stars that are burning helium in their cores. These stars are considered to be the progeny of red giant branch (RGB) stars (Hoyle \& Schwarzschild 1955). To locate on different positions of $\mathrm{HB}, \mathrm{RGB}$ stars need to lose different envelope masses before or during helium core flash is taking place (Catelan 2009). Some stars could settle on the red HB (RHB) positions in the colour-magnitude diagram (CMD) of GCs after losing a few envelope masses, while other stars may occupy the blue HB (BHB) or extreme HB (EHB) positions due to which they lose most of or nearly the whole envelope masses on the RGB stage. However, the physical mechanism of mass loss for RGB stars in GCs is still unclear (Willson 2000; Dupree et al. 2009).

In the late 1990s, a special kind of hot EHB stars are found in

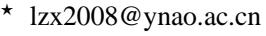

† gzhao@nao.cas.cn

† zhanwenhan@ynao.ac.cn
}

some massive GCs (e.g., NGC 2808, $\omega$ Cen; Whitney et al. 1998; D'Cruz et al. 2000), which are the so called blue hook (BHk) stars. These stars present very high temperatures (e.g., $T_{\text {eff }}>32000 \mathrm{~K}$; Moni Bidin et al. 2012) and very faint luminosity when compared with normal EHB stars in GCs. Therefore, BHk stars can not be predicted by canonical stellar evolution models, and their formation mechanism is still unclear. So far, several formation scenarios are proposed for BHk stars in GCs (see Heber 2016 for a recent review). D'Antona et al. (2010) proposed that BHk star in $\omega$ Cen could be the progeny of blue main-sequence (MS) stars that belong to the second generations in this GC (also see Lee 2005). These stars would undergo an extra mixing during the RGB stage, thus present very high helium abundance in their surfaces (e.g., up to $Y \approx 0.8$ ). After helium ignites in their cores, these stars locate on very blue and faint $\mathrm{HB}$ position and become BHk stars in GCs. On the other hand, Brown et al. (2001) suggested that BHk stars could be produced through late hot flash process on the white dwarf (WD) cooling curve (also see Castellani \& Castellani 1993; D'Cruz et al. 1996; Brown et al. 2010, 2012). In this scenario, low mass stars in GCs experience huge mass loss on the RGB and undergo helium 
core flash, instead of at the RGB tip, on the way towards to WD stage (early hot flash; Brown et al. 2001; Cassisi et al. 2003; Miller Bertolami et al. 2008) or on the WD cooling curve (late hot flash; Brown et al. 2001; Cassisi et al. 2003; Miller Bertolami et al. 2008). During the late hot flash, the internal convection can permeate the thin hydrogen-enriched envelope and lead to helium and carbon enhancement in the surface. Therefore, when settling on the zero age horizontal branch (ZAHB), these stars that experience late hot flash could be hotter and fainter than normal EHB stars in GCs.

Recently, more and more pieces of evidence both from photometry and spectroscopy support that multiple populations could be a universal phenomenon in most of the Galactic GCs (Piotto et al. 2007; Gratton Carratta \& Bragaglia 2012; Gratton et al. 2013, 2014; Milone 2015) and this phenomenon is considered to be closely correlated with helium enrichment in GCs (D'Antona \& Caloi 2008; Marino et al. 2014; Milone 2015; but see Jiang et al. 2014 for an alternative solution). If this is the case, stars in GCs would belong to different populations that present different helium abundances, and BHk stars would be the progeny of heliumenriched populations that belong to the second generation stars in GCs (D'Antona et al. 2002; Brown et al. 2012). Following this scenario, Tailo et al. (2015) also studied the effects of rapidly rotating second generation stars on the formation of BHk stars in GCs. They found that an increase of helium core mass up to $0.04 M_{\odot}$ is required to solve the luminosity range problem for BHk stars in $\omega$ Cen.

Lei et al. (2015, hereafter Paper I) followed the late hot flash scenario and proposed that tidally enhanced stellar wind in binary evolution (Tout \& Eggleton 1988) is a possible formation channel for BHk stars in GCs. This kind of wind could provide huge mass loss on the RGB stage naturally, which is needed in late hot flash scenario (Brown et al. 2001). Their results indicated that binaries could produce BHk stars under tidally enhanced stellar wind and it may play important roles in the formation of BHk stars in some GCs. However, Paper I did not study the effects of other input parameters on their results, such as metallicity, mass ratio of primary to secondary, tidal enhancement efficiency, and helium abundance, etc. These parameters would influence the evolution of binary systems (e.g., mass loss of the primary, stellar mass, helium core mass, luminosity, etc), thus may have influences on the formation of BHk stars in GCs. As a further study for Paper I, to investigate the role of binaries in the formation of BHk stars, we studied in this paper the effects of metallicity, mass ratio, tidal enhancement efficiency and helium abundance on the binary evolution by considering tidally enhanced stellar wind into binary evolution, thus studied their effects on the formation of BHk stars in GCs. The structure of this paper is organized as follows: In Section 2, we describe the models and method used in this study; results are given in Section 3; and finally, discussion and conclusions are given in Sections 4 and 5 respectively.

\section{METHODOLOGY}

As in Paper I, we use equation (1) to describe the tidally enhanced stellar wind in binary evolution, which was first suggested by Tout \& Eggleton (1988).

$\dot{M}=-\eta 4 \times 10^{-13}(R L / M)\left\{1+B_{\mathrm{w}} \times \min \left[\left(R / R_{\mathrm{L}}\right)^{6}, 1 / 2^{6}\right]\right\}$,

where $\eta$ is the Reimers mass-loss efficiency (Reimers 1975), and $B_{\mathrm{w}}$ is the efficiency of tidal enhancement for the stellar wind. Here $R, L$, and $M$ are the radius, luminosity and mass of the primary star in solar units. Equation (1) was added into detailed stellar evolution code, Modules for Experiments in Stellar Astrophysics (MESA, version 6208; Paxton et al. 2011, 2013, 2015) to study its effects on the mass loss of primary during RGB evolution stage.

We use the binary module in MESA to evolve the primary star in a binary system from zero-age main sequence (ZAMS) to WD cooling curve. Since the stellar wind on the RGB stage could be tidally enhanced by the secondary stars, some of the primary stars could lose nearly the whole envelope mass and evolve off RGB tip, then experience a late hot helium flash on WD cooling curve (Castellani \& Castellani 1993; D'Cruz et al. 1996; Brown et al. 2001). Due to the very thin hydrogen envelope, internal convection mixing triggered by helium core flash is able to penetrate into the surface and lead to helium and carbon enhancement. When settling on $\mathrm{HB}$, these stars could present higher temperatures and faint luminosity than normal EHB star.

In this study, the default values of input physics in MESA are used except for the opacity tables. OPAL type II tables are used in our model calculations that would be more suitable for helium burning stars (see Paper I). The Reimers mass-loss efficiency, $\eta$, is set to 0.45 (Renzini \& Fusi Pecci 1988; McDonald \& Zijlstra 2015). All the input parameters used in this study are listed in Table 1. Columns 1-3 gives the model number, stellar mass of the primary star at ZAMS ( $M_{\text {ZAMS }}$ ) and initial helium abundance. The value of $M_{\text {ZAMS }}$ for each model adopted here corresponds to an age of about 12 Gyr at RGB tip. Columns 4-7 present some critical orbital periods for binary models, which will be introduced in next paragraph. Different from Paper I, we adopted three values of metallicity (i.e., $Z=0.003,0.001$ and 0.0003 ) in this study to investigate its effects on the final results. For each metallicity, the mass ratio of primary to secondary (i.e., $q$ ) and tidal enhancement efficiency (i.e., $B_{\mathrm{w}}$ ) is set to 1.6 and 10000 , respectively. These models are labelled by I, II and III in Table 1. Excluding $q=1.6$, we also adopted other two values of mass ratio in the study (i.e., $q=1.2$ and 2.4) for metallicity $Z=0.001$. These two models are labelled by IV and V in Table 1 . Moreover, other two values of $B_{\mathrm{w}}$ i.e., 5000 and 1000) were used in this study for the models with $Z=0.001$ and $q=1.6$ to investigate its effects on our final results, and these models are labelled by VI and VII in Table 1. For each model, four values of initial helium abundance were used, i.e., $Y=0.24,0.28,0.32$ and 0.40 . There are a total of 28 sets of binary models combined with different input parameters from model I to VII.

In tidally enhanced stellar-wind model (Lei et al. 2013a, b; also see Han, Chen \& Lei 2010; Han et al. 2012; Han \& Lei 2014), the mass-loss of primary star in a binary system is determined by the initial orbital period. Thus, the primary stars in binary systems with long orbital period may locate on RHB position due to little mass loss on the RGB stage, while primary stars in binary systems with shorter periods would lose much envelope masses and settle on hotter HB positions or even fail to ignite helium in their cores and become a helium WD finally. For each set of binary model list in Table 1, we adopted different initial orbital periods in binary evolution to find out what kind of initial periods in binary would produce BHk stars. From columns 4 to 7 of Table 1, we list four critical periods for each binary model that are important in our calculations. Specifically, the initial orbital periods listed in column 4 (e.g., labelled by $P_{1}$ ) represent the minimum periods for primary stars to experience normal helium core flash at RGB tip. If the initial orbital period is shorter than this one, the primary star would lose much envelope mass on the RGB and experience early or late hot flash before locating on HB. On the other hand, initial periods listed in column 5 (e.g., labelled by $P_{2}$ ) for each set of model 
Table 1. Main input parameters used in the study. The masses of primary stars at ZAMS in each set correspond to an age of about 12 Gyr at RGB tip.

\begin{tabular}{|c|c|c|c|c|c|c|}
\hline Model & $M_{\mathrm{ZAMS}} / M_{\odot}$ & $Y$ & $P_{1} / \mathrm{d}$ & $P_{2} / \mathrm{d}$ & $P_{3} / \mathrm{d}$ & $P_{4} / \mathrm{d}$ \\
\hline I & $Z=0.003$ & $q=1.6$ & $B_{\mathrm{w}}=10000$ & & & \\
\hline set 1 & 0.87 & 0.24 & 2850 & 2700 & 2160 & 2150 \\
\hline set 2 & 0.81 & 0.28 & 3100 & 2850 & 2230 & 2220 \\
\hline set 3 & 0.75 & 0.32 & 3500 & 3100 & 2330 & 2320 \\
\hline set 4 & 0.64 & 0.40 & 10000 & 4600 & 2680 & 2670 \\
\hline II & $Z=0.001$, & $q=1.6$ & $B_{\mathrm{w}}=10000$ & & & \\
\hline set 5 & 0.83 & 0.24 & 2200 & 2000 & 1610 & 1600 \\
\hline set 6 & 0.77 & 0.28 & 2300 & 2150 & 1670 & 1660 \\
\hline set 7 & 0.72 & 0.32 & 2600 & 2260 & 1700 & 1690 \\
\hline set 8 & 0.62 & 0.40 & 10000 & 3100 & 1890 & 1880 \\
\hline III & $Z=0.0003$ & $q=1.6$ & $B_{\mathrm{w}}=10000$ & & & \\
\hline set 9 & 0.81 & 0.24 & 1700 & 1560 & 1300 & 1290 \\
\hline set 10 & 0.758 & 0.28 & 1800 & 1650 & 1320 & 1310 \\
\hline set 11 & 0.70 & 0.32 & 2050 & 1800 & 1400 & 1390 \\
\hline set 12 & 0.61 & 0.40 & 10000 & 2350 & 1500 & 1490 \\
\hline IV & $Z=0.001$, & $q=1.2$ & $B_{\mathrm{w}}=10000$ & & & \\
\hline set 13 & 0.83 & 0.24 & 2250 & 2150 & 1730 & 1720 \\
\hline set 14 & 0.77 & 0.28 & 2600 & 2300 & 1800 & 1790 \\
\hline set 15 & 0.72 & 0.32 & 2650 & 2400 & 1820 & 1810 \\
\hline set 16 & 0.62 & 0.40 & 10000 & 3200 & 2020 & 2010 \\
\hline $\mathrm{V}$ & $Z=0.001$, & $q=2.4$ & $B_{\mathrm{w}}=10000$ & & & \\
\hline set 17 & 0.83 & 0.24 & 1900 & 1750 & 1440 & 1430 \\
\hline set 18 & 0.77 & 0.28 & 2100 & 1900 & 1500 & 1490 \\
\hline set 19 & 0.72 & 0.32 & 2350 & 2050 & 1530 & 1520 \\
\hline set 20 & 0.62 & 0.40 & 10000 & 2750 & 1720 & 1710 \\
\hline VI & $Z=0.001$, & $q=1.6$ & $B_{\mathrm{w}}=5000$ & & & \\
\hline set 21 & 0.83 & 0.24 & 1800 & 1680 & 1350 & 1340 \\
\hline set 22 & 0.77 & 0.28 & 1960 & 1780 & 1410 & 1400 \\
\hline set 23 & 0.72 & 0.32 & 2100 & 1900 & 1430 & 1420 \\
\hline set 24 & 0.62 & 0.40 & 10000 & 2700 & 1590 & 1580 \\
\hline VII & $Z=0.001$, & $q=1.6$ & $B_{\mathrm{w}}=1000$ & & & \\
\hline set 25 & 0.83 & 0.24 & 1200 & 1130 & 910 & 900 \\
\hline set 26 & 0.77 & 0.28 & 1350 & 1200 & 940 & 930 \\
\hline set 27 & 0.72 & 0.32 & 1500 & 1260 & 960 & 950 \\
\hline set 28 & 0.62 & 0.40 & 10000 & 1750 & 1070 & 1060 \\
\hline
\end{tabular}

present the minimum periods for primary stars to undergo early hot flash, while the periods labeled by $P_{3}$ in column 6 denote the minimum periods for the primary stars to experience late hot flash. If the initial orbital period of a binary is shorter than $P_{3}$ (e.g., periods list in the last column of Table 1 , labeled by $P_{4}$ ), the primary star may lose too much envelope mass on the RGB and fail to ignite helium in its core, then dies as a helium WD (see Fig. 1).

In Table 1, for all the binary models with the highest helium abundance of $Y=0.40$ (e.g., set 4, 8, 12, 16, 20, 24 and 28), the minimum orbital periods for primary stars to experience normal helium core flash at the RGB tip (i.e., the orbital periods labeled by $P_{1}$ in Table 1) are set to $10000 \mathrm{~d}$. This is because these models present the highest helium abundance, thus the lowest stellar mass at ZAMS. Therefore, even given a very long initial orbital period, e.g., $P=10000 \mathrm{~d}$, for which the two components in these binaries could be considered as two single stars, the primary star still experience an early hot flash instead of a normal helium flash at the RGB tip [see panel (m) in Fig. 1]. These stars would become hot
EHB stars rather than BHk stars after helium core flash, and it is beyond the range of this study.

\section{RESULTS}

In the tidally enhanced stellar-wind model, we evolve the primary star from ZAMS to WD, and obtain the evolution parameters (e.g., effective temperatures, gravities, luminosity and surface chemical composition etc.). These parameters could be compared with observations directly or with observations after translation.

\subsection{Evolution tracks}

Fig. 1 shows the evolution tracks of primary stars from ZAMS to WD for the binary systems of model II list in Table 1 (e.g., sets 5-8). Note that the evolution tracks for other models list in Table 1 are not presented, because they are very similar with the ones we showed here. Specifically, panels (a)-(d) in Fig. 1 show the evolution tracks 


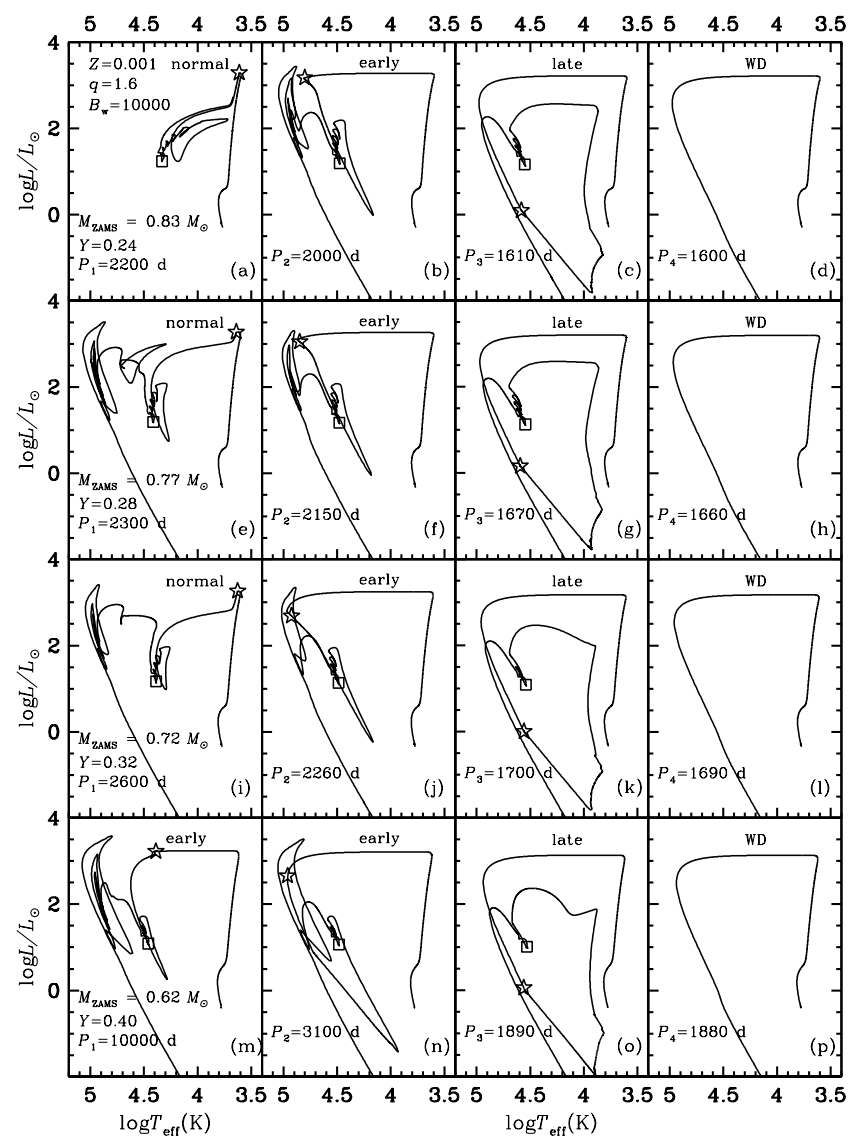

Figure 1. The evolution tracks for the primary stars for binaries list in model II of Table 1 (e.g., sets 5-8). The pentacles in each panel represent the positions where the primary helium core flash takes place, while the open squares denote ZAHB positions for each track. The initial orbital periods for each binary system are labelled in each panel.

for binary models with different initial orbital periods labelled by set 5 in Table 1 (i.e., $M_{\text {ZAMS }}=0.83 M_{\odot} ; Y=0.24$ ), panels (e)-(h) show the evolution tracks of set 6 (i.e., $M_{\mathrm{ZAMS}}=0.77 M_{\odot} ; Y=0.28$ ), panels (i)-(l) present evolution tracks of set 7 (i.e., $M_{\text {ZAMS }}=0.72 M_{\odot}$; $Y=0.32$ ), while panels $(\mathrm{m})$-(p) present evolution tracks of set 8 (i.e., $\left.M_{\text {ZAMS }}=0.62 M_{\odot} ; Y=0.40\right)$. The initial orbital periods are also labeled in each panel. In Fig. 1, pentacles denote the positions on $\mathrm{H}-\mathrm{R}$ diagram where helium core flash is taking place, while open squares denote the ZAHB positions for each model. The label 'normal' in Fig. 1 means the primary star experience normal helium core flash at the RGB tip; 'early' means the primary star experience helium core flash on the way towards WD (i.e., early hot flash); 'late' means that helium core flash is taking place on the WD cooling curve (i.e., late hot flash); while 'WD' means the primary stars lose too much envelope masses on the RGB stage to ignite helium burning and become helium WDs finally.

As we discussed in Paper I (also see Brown et al. 2001, 2010, 2012), early hot flash process could produce hot EHB stars rather than BHk stars (also see Paper I), since the internal convection mixing triggered by helium core flash can not reach the hydrogenenriched surface and change the surface chemical composition. On the other hand, due to the very thin hydrogen-enriched envelope, convection mixing in late hot flash process could penetrate the surface and engulf hydrogen into hotter interior. Then, a helium and carbon-enhanced envelope is left, which makes a star present higher temperature and faint luminosity than normal EHB stars when settling on ZAHB. As we described in Section 2, the initial orbital periods labelled by $P_{2}$ in Table 1 denote the minimum periods for early hot flash, below which the primary star would experience late hot flash due to losing much envelope mass, while initial orbital periods labelled by $P_{3}$ in Table 1 represent the minimum periods for late hot flash, below which the primary star would fail to ignite helium burning in its core and die as a helium WD due to losing too much envelope mass. It means that, for each set of binary model list in Table 1, the primary stars would experience late hot helium flash and become BHk stars if the initial orbital period is between $P_{2}$ and $P_{3}$ (i.e., $P_{3} \leqslant P<P_{2}$ ). One can see from Fig. 1 that, even though their initial helium abundances are very different, all sets of binary models could produce BHk stars within different period range (e.g., $1610 \leqslant P<2000$ for $Y=0.24 ; 1670 \leqslant P<2150$ for $Y=0.28 ; 1700 \leqslant$ $P<2260$ for $Y=0.32 ; 1890 \leqslant P<3100$ for $Y=0.40)$. The ranges of orbital periods to produce BHk stars for other binary models with different input parameters are shown in Table 1. We will discuss the effects of the input parameters on our results in Section 4, including metallicity, mass ratio, tidal enhancement efficiency and helium abundance.

\subsection{Evolution parameters at ZAHB}

We also presented the evolution parameters at ZAHB for the primary stars presented in Fig. 1. From the left to the right of Table 2 , it gives initial orbital period (in days), stellar mass at ZAHB $\left(M_{\mathrm{ZAHB}}\right)$, effective temperature, gravity, surface hydrogen, surface helium, surface carbon and flash status, respectively. Flash status in Table 2 denote what kind of helium core flash does the primary star experience before locating on ZAHB, and the labels 'normal', 'early' and 'late' have the same meaning as we described in Fig. 1. The evolution parameters for the tracks that have failed to ignite helium burning in their cores are not listed in Table 2 .

As we discussed in Paper I, the surface chemical abundances are not changed by internal flash mixing in the 'normal' and 'early' models. However, due to losing more envelope mass on the RGB stage, 'early' models present higher effective temperatures than 'normal' models by about several thousands of $\mathrm{K}$ at ZAHB. On the other hand, 'late' models experience late hot flash when descending WD cooling curve. They present the highest effective temperatures and helium and carbon enhancement in the surface due to internal flash mixing. One can see from Table 2, with initial helium abundance increasing from $Y=0.24$ to 0.40 , that the effective temperatures and luminosity of 'late' models in each set model decrease slowly, while their gravities increase gradually. This is because that, for the 'late' models, core helium burning provide the mainly energies released at HB stage (e.g., the hydrogen-enriched envelope is too thin to ignite hydrogen burning during HB stage; Brown et al. 2001; Cassisi et al. 2003). However, for higher initial helium abundance, the stellar masses at ZAMS ( $\left.M_{\text {ZAMS }}\right)$ are smaller if stars have same ages, thus these stars have smaller helium core mass when settling on ZAHB, and present lower temperatures and luminosity but larger gravities than the stars with lower initial helium abundance.

One can also see from Table 2 that the 'normal' and 'early' models show normal hydrogen, helium and carbon abundance in the surface, because the flash progress does not change the chemical composition in the surface. However, for the 'late' models (e.g., the model with $P=1610$ d in set 5 of Table 2), flash triggered mixing could enhance helium up to 0.9701 and carbon up to 0.0107 in the surface. Miller Bertolami et al. (2008) also studied the late hot flash scenario for the formation of He-rich subdwarf stars with 
Table 2. The evolution parameters for the primary stars of model II in Table 1 when settling on ZAHB. From the left to the right of the table, it presents the initial orbital periods (in days), stellar mass at ZAHB ( $\left.M_{\mathrm{ZAHB}}\right)$, effective temperature at ZAHB, luminosity at ZAHB, gravity at ZAHB, the surface hydrogen, helium and carbon at ZAHB, and flash status. Flash status means what kind of helium flash does the primary star experience before settling on ZAHB. See the text for details.

\begin{tabular}{|c|c|c|c|c|c|c|c|c|}
\hline$P / \mathrm{d}$ & $M_{\mathrm{ZAHB}} / M_{\odot}$ & $\log T_{\text {eff }}$ & $\log L / L_{\odot}$ & $\log g$ & $X_{\text {surf }}$ (by mass) & $Y_{\text {surf }}$ (by mass) & $C_{\text {surf }}$ (by mass) & Flash status \\
\hline set 5 & $M_{\mathrm{ZAMS}}=0.83 M_{\odot}$ & $Y=0.24$ & & & & & & \\
\hline 2200 & 0.5036 & 4.3323 & 1.2393 & 5.1833 & $7.4431 \times 10^{-1}$ & $2.5317 \times 10^{-1}$ & $1.6144 \times 10^{-4}$ & normal \\
\hline 2000 & 0.4829 & 4.4756 & 1.1941 & 5.7837 & $7.4431 \times 10^{-1}$ & $2.5317 \times 10^{-1}$ & $1.6161 \times 10^{-4}$ & early \\
\hline 1610 & 0.4702 & 4.5498 & 1.1593 & 6.1035 & $1.3548 \times 10^{-3}$ & $9.7006 \times 10^{-1}$ & $1.0676 \times 10^{-2}$ & late \\
\hline set 6 & $M_{\mathrm{ZAMS}}=0.77 M_{\odot}$, & $Y=0.28$ & & & & & & \\
\hline 2300 & 0.4822 & 4.4128 & 1.1881 & 5.5379 & $7.0702 \times 10^{-1}$ & $2.9043 \times 10^{-1}$ & $1.6325 \times 10^{-4}$ & normal \\
\hline 2150 & 0.4752 & 4.4792 & 1.1696 & 5.8157 & $7.0702 \times 10^{-1}$ & $2.9043 \times 10^{-1}$ & $1.6325 \times 10^{-4}$ & early \\
\hline 1670 & 0.4620 & 4.5454 & 1.1234 & 6.1140 & $1.5369 \times 10^{-3}$ & $9.7103 \times 10^{-1}$ & $1.0145 \times 10^{-2}$ & late \\
\hline set 7 & $M_{\mathrm{ZAMS}}=0.72 M_{\odot}$, & $Y=0.32$ & & & & & & \\
\hline 2600 & 0.4811 & 4.3869 & 1.1770 & 5.4444 & $6.6936 \times 10^{-1}$ & $3.2820 \times 10^{-1}$ & $1.6487 \times 10^{-4}$ & normal \\
\hline 2260 & 0.4673 & 4.4859 & 1.1384 & 5.8662 & $6.6936 \times 10^{-1}$ & $3.2820 \times 10^{-1}$ & $1.6487 \times 10^{-4}$ & early \\
\hline 1700 & 0.4535 & 4.5401 & 1.0881 & 6.1204 & $4.8556 \times 10^{-3}$ & $9.6951 \times 10^{-1}$ & $1.0017 \times 10^{-2}$ & late \\
\hline set 8 & $M_{\mathrm{ZAMS}}=0.62 M_{\odot}$, & $Y=0.40$ & & & & & & \\
\hline 10000 & 0.4559 & 4.4587 & 1.0871 & 5.7981 & $5.9338 \times 10^{-1}$ & $4.0440 \times 10^{-1}$ & $1.7109 \times 10^{-4}$ & early \\
\hline 3100 & 0.4503 & 4.4821 & 1.0635 & 5.9099 & $5.9338 \times 10^{-1}$ & $4.0440 \times 10^{-1}$ & $1.7109 \times 10^{-4}$ & early \\
\hline 1890 & 0.4365 & 4.5293 & 1.0101 & 6.1385 & $1.0158 \times 10^{-2}$ & $9.6598 \times 10^{-1}$ & $1.1008 \times 10^{-2}$ & late \\
\hline
\end{tabular}

a wide range of metallicities and physical assumptions. For their model with $Z=0.001$, they found a helium enhancement in the surface up to 0.9666 and carbon enhancement up to 0.0107 . Their results are very similar with our results found here. Furthermore, Miller Bertolami also found a transition type of late hot flash which is called the 'shallow' mixing cases (also see Lanz et al. 2004). In this kind of flash, the hydrogen envelope is mixed only with the convective shell in the outer part of the core, thus none of the hydrogen captured by the convective shell will be burned. Therefore, the surface helium abundance is lower than the case that experience 'deep' mixing (e.g., $Y=0.7475$ in the 'shallow' model with $Z=0.001$ listed in table 3 in Miller Bertolami et al. 2008 ). However, this kind of hot flash is rare for low metallicities (Lanz et al. 2004; Miller Betrolami et al. 2008). Latour et al. (2014) obtained the fundamental parameters (e.g., effective temperature, gravity, helium and carbon abundance) for 38 hot subdwarf stars in $\omega$ Cen. They found that, for some He-rich hot subdwarf stars, the surface helium enhancement could reach 0.92 and carbon enhancement could reach 0.015 . Their results are consistent with ours presented here. Moreover Latour et al. (2014) also found a positive correlation between helium and carbon enhancement among He-rich subdwarf stars in their sample, which support the late hot flash scenario for the formation of $\mathrm{BHk}$ stars in $\omega$ Cen.

\subsection{Comparison with observation in $T_{\text {eff }}-\log g$ diagram}

BHk stars were first found in massive GC NGC 2808 and $\omega$ Cen (Whitney et al. 1998; D'Cruz et al. 2000). NGC 2808 is a typical GC that hosts splitting MS and extended HB (Piotto et al. 2002, 2007). Its metallicity is about $Z=0.0014$ or $[\mathrm{Fe} / \mathrm{H}]=-1.15$ (Harris 1996, version 2003) and its age is in the range of 10.4-12.9 Gyr (Gratton et al. 2010). $\omega$ Cen is a complex system among GCs, and some researchers considered it as the surviving nucleus of a dwarf galaxy captured and disrupted by Milky Way several Gyr ago (Dinescu et al. 1999; Bellazzini et al. 2008; Marconi et al. 2014). It presents two parallel MS and at least five stellar populations in sub giant branch ( Bedin et al. 2004; Piotto et al. 2005; Villanova et al. 2007, 2014). Furthermore, $\omega$ Cen cover a wide range of metallicity,

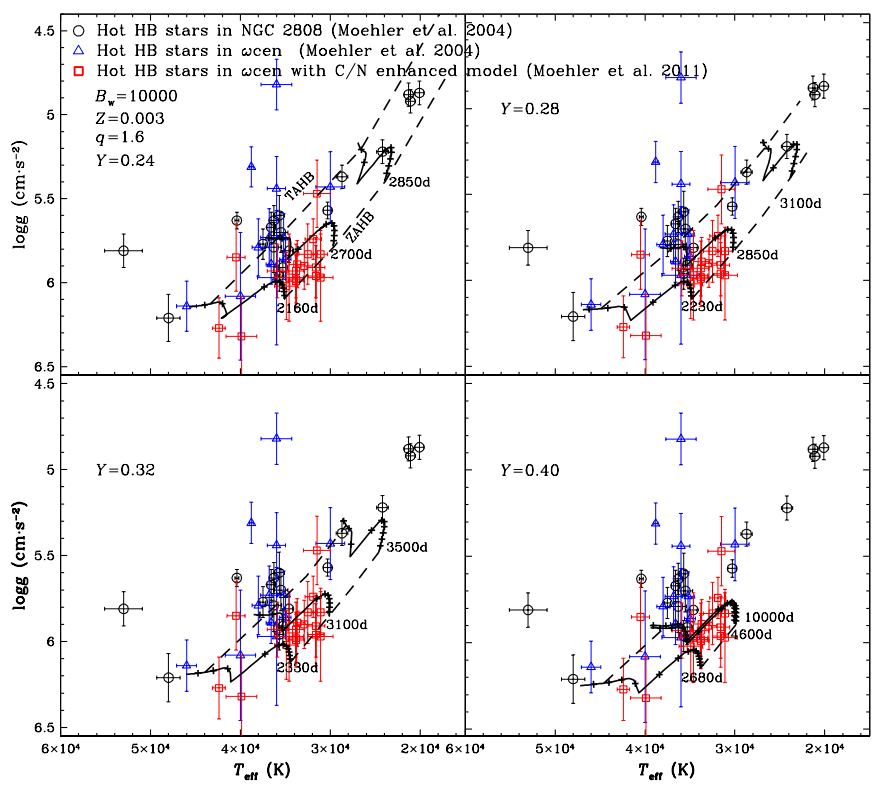

Figure 2. Comparison between the results of model I and observations in the $T_{\text {eff }}{ }^{-}$ $\log g$ plane of NGC 2808 and $\omega$ Cen . The input parameters adopted in model I are labeled, and the time interval between two adjacent + symbols in each track is $10^{7} \mathrm{yr}$

e.g., $-2.2 \leqslant[\mathrm{Fe} / \mathrm{H}] \leqslant-0.6$ (Johnson \& Pilachowski 2010; Villanova et al. 2014). Since the parameters of NGC 2808 are very similar with the values we adopted here, we compared our results with this GC in the $T_{\text {eff }}-\log g$ diagram. Though $\omega$ Cen cover a wide range of metallicity, we compared our results with this GC in $T_{\text {eff }}-\log g$ diagram as well, because we also adopted three different values of metallicity in our calculations, e.g., 0.003 or $[\mathrm{Fe} / \mathrm{H}]=-0.8,0.001$ or $[\mathrm{Fe} / \mathrm{H}]=-1.3$ and 0.0003 or $[\mathrm{Fe} / \mathrm{H}]=-1.8$, and these values are in the metallicity range of $\omega$ Cen.

Figs 2-8 present the comparison between our model calculation results (i.e., evolution tracks at $\mathrm{HB}$ stage from models I to VII listed in Table 1) and observation in $T_{\text {eff }}-\log g$ diagram of GC NGC 
2808 and $\omega$ Cen. The black open circles in these figures represent BHk stars and hot EHB stars in GC NGC 2808, and the blue open triangles represent BHk stars and hot EHB stars in $\omega$ Cen. These spectral parameter data for the hot HB stars in NGC 2808 and $\omega$ Cen are from Moehler et al. (2004), while the red open squares in these figures are the hot He-rich HB stars in $\omega$ Cen studied by Moehler et al. (2011). The spectral parameters of these hot stars are obtained by using $\mathrm{C} / \mathrm{N}$-enhanced model atmospheres with mass fraction of $3 \%$ and $1 \%$ for carbon and nitrogen, which is predicted by the late hot flash scenario (Lanz et al. 2004; Miller Bertolami et al. 2008). When $\mathrm{C} / \mathrm{N}$-enhanced model atmospheres are used, the obtained gravities of the hot HB stars would be higher than the values obtained without $\mathrm{C} / \mathrm{N}$-enhanced model atmospheres by an average of about 0.1 dex (see table 4 in Moehler et al. 2011). The dashed line at the bottom labeled by 'ZAHB' in each panel of Figs 2-8 represent the ZAHB positions for our evolution tracks, while the dashed line at the top labelled by 'TAHB' represent the terminal age HB (TAHB), which is defined as the stage when central helium abundance dropped below $1 \times 10^{-4}$. The black solid curves in each panel of Fig. 2 to 8 denote the evolution tracks at HB stage for the primary stars listed in table 1 . The value of initial orbital periods (in days) are also labeled nearby the tracks, and the input parameters for these models are also labelled.

Fig 2 shows the comparison between the results of model I list in Table 1 and the observation. These models have the input parameters of $Z=0.003, q=1.6$ and $B_{\mathrm{w}}=10000$. The initial helium of the binary models from upper left to bottom right panel is $Y=0.24$, $0.28,0.32$ and 0.40 , respectively. One can see that in the upper left panel (i.e., $Y=0.24$ ), most of the BHk stars (e.g., $T_{\text {eff }}>32000 \mathrm{~K}$; Moni bidin et al. 2012) in NGC 2808 and $\omega$ Cen from Moehler et al. (2004) locate in the region predicted by our models. However, the gravities of BHk stars predicted by our models seem to be a little larger than the values obtained by Moehler et al. (2004). This discrepancy between model calculations and observations becomes more evident when initial helium abundance becomes higher (see other three panels in fig 2 with $Y=0.28,0.32,0.40$ ). Especially for $Y=0.40$ (bottom right panel in fig 2), one can see that most of the BHk stars from Moehler et al. (2004), e.g., black open circles and black open triangles, locate out of the HB region defined by ZAHB and TAHB line. A possible explanation for this discrepancy between model calculations and observations would be that the late hot flash scenario predicted carbon and nitrogen enhancement in the surface of BHk stars (e.g., 3\% and 1\% for carbon and nitrogen by mass in Lanz et al. 2004). However, this enhancement was not considered in the atmosphere model used to obtain the atmosphere parameters of BHk stars in NGC 2808 and $\omega$ Cen in Moehler et al. (2004). On the other hand, Moehler et al. (2011) did consider the carbon and nitrogen enhancement in the atmosphere model when obtaining the parameters of some BHk stars in $\omega$ Cen. These BHk stars are denoted by red open squares in figs 2-8. One can see clearly in fig 2 that when $\mathrm{C} / \mathrm{N}$-enhanced model atmospheres are considered, most of the BHk sample stars in Moehler et al. (2011) are well predicted by our late hot flash models, regardless of the helium abundance adopted, including the highest value of $Y=0.40$. This is also the case for figs 3-8. Thus, if carbon and nitrogen enhancement is considered, one would expect a better agreement between our calculation results and the BHk stars in NGC 2808 and $\omega$ Cen from Moehler et al. (2004).

One can see from figs 2-8, that under the tidally enhanced stellar wind, all the binary models from I to VII could produce BHk stars, though the input parameters are different with each other. For figs 2-4, which correspond to models I , II and III in Table

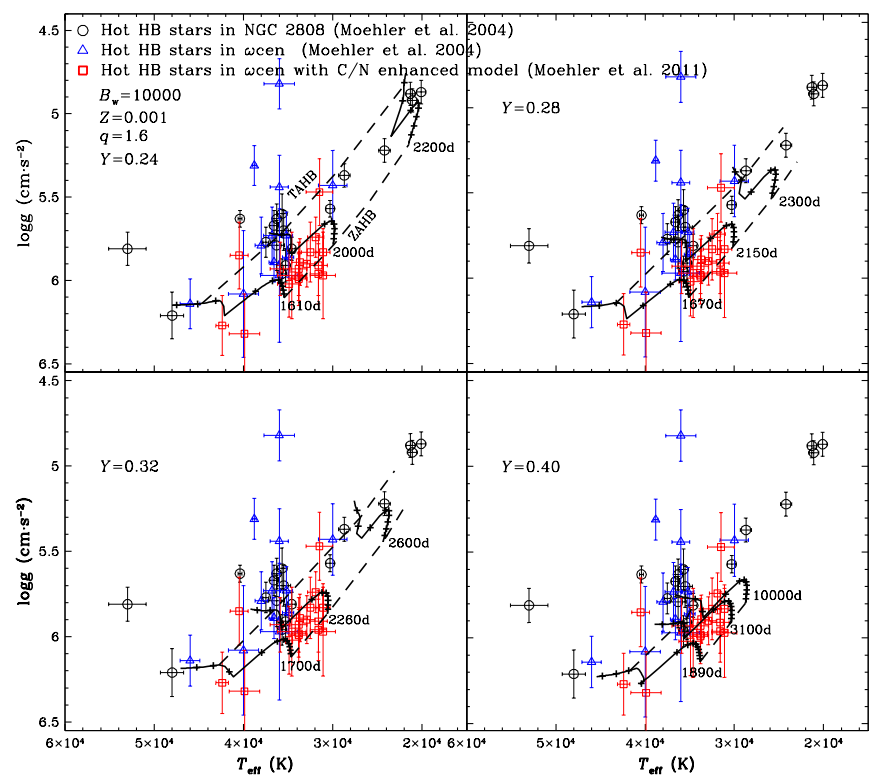

Figure 3. The same as Fig. 2, but for the comparison between observations and model II.

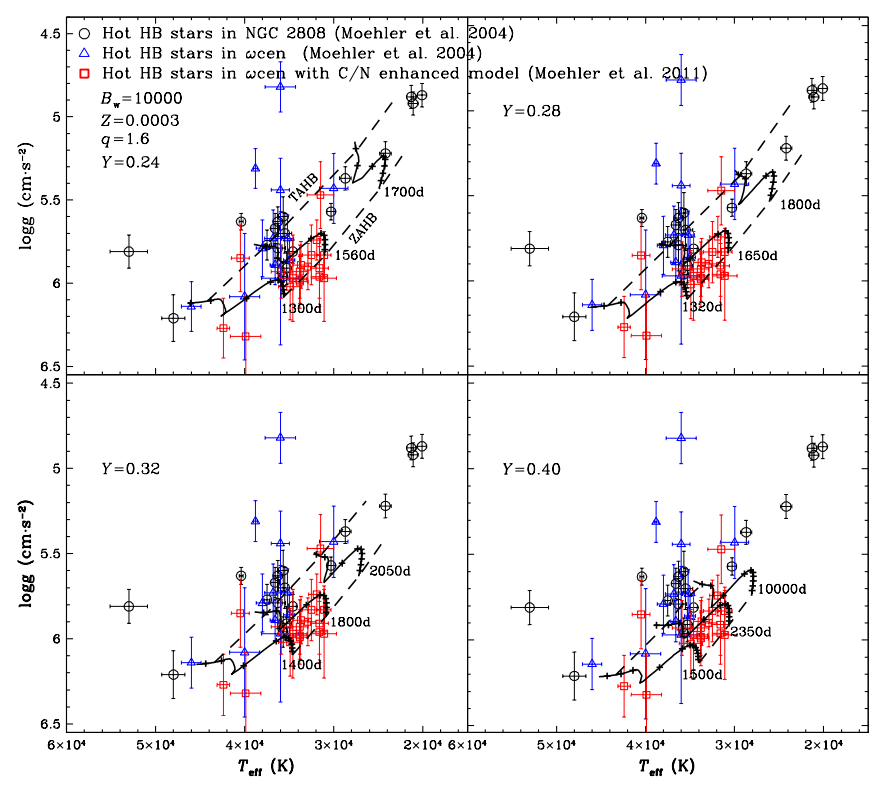

Figure 4. The same as Fig. 2, but for the comparison between observations and model III.

1 , the binaries in these models have the same mass ratio of primary to secondary (i.e., $q=1.6$ ) and tidal enhancement efficiency (e.g., $B_{\mathrm{w}}=10000$ ), but with different metallicities (e.g., $Z=0.003,001$ and 0.0003 for model I, II and III, respectively); figs 3, 5 and 6, which correspond to models II, IV and V, present the same metallicity (i.e., $Z=0.001$ ) and tidal enhancement efficiency (i.e., $B_{\mathrm{w}}=10000$ ), but with different mass ratios (e.g., $q=1.6,1.2$ and 2.4 for models II, IV and V respectively). While figs 3, 7 and 8, which correspond to models II, VI and VII, have the same metallicity (i.e., $Z=0.001$ ) and mass ratio (i.e., $q=1.6$ ), but with different tidal enhancement efficiency (e.g., $B_{\mathrm{w}}=10000,1000$ and 5000 respectively). We will discuss the effects of the various input parameters on our results in next section. 


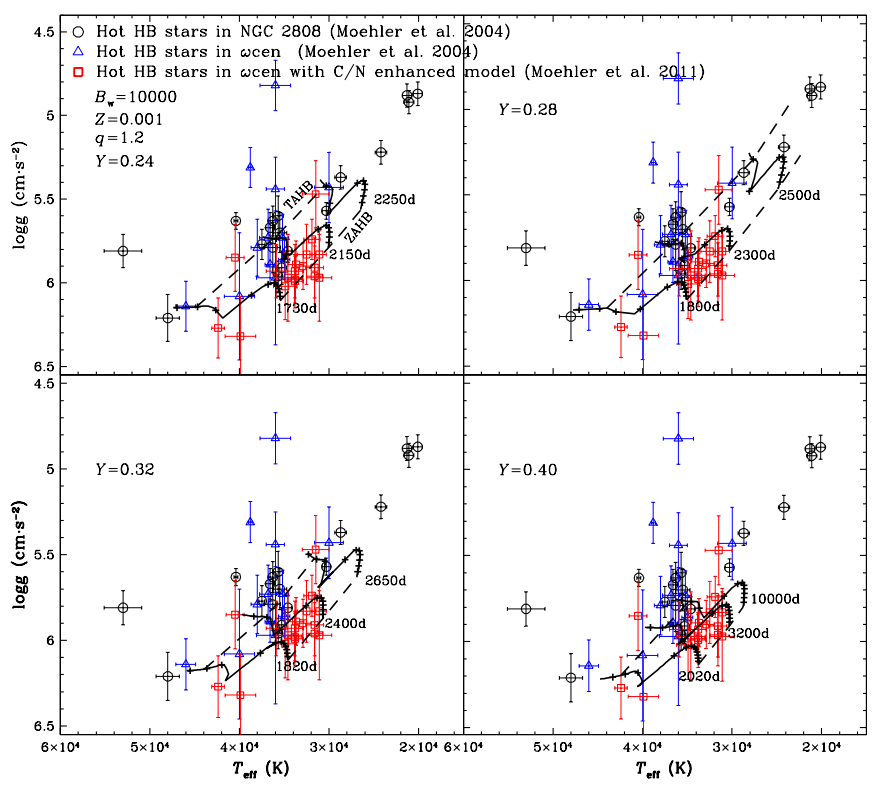

Figure 5. The same as Fig. 2, but for the comparison between observations and model IV.

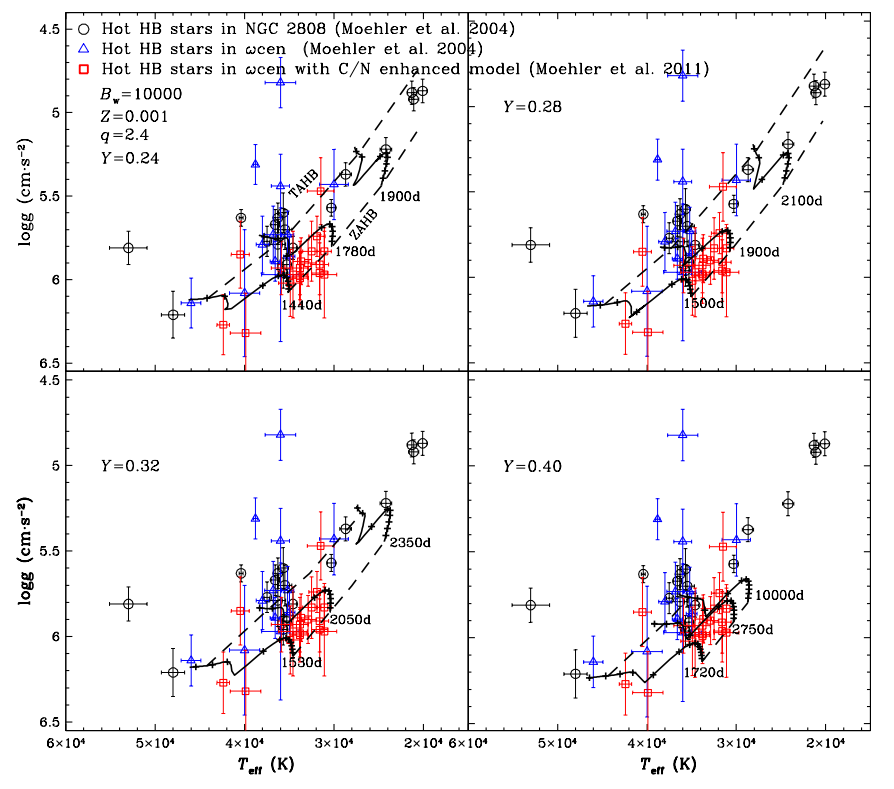

Figure 6. The same as Fig. 2, but for the comparison between observations and model V.

\section{DISCUSSION}

In the tidally enhanced stellar-wind model, the primary star would lose huge envelope mass during RGB stage and experience late hot helium flash on the WD cooling curve to become a BHk star. Though the mass loss of the primary stars in this scenario depends on the initial orbital periods of the binaries (Paper I, also see the discussion in Section 2 of this study), other input parameters such as metallicity, mass ratio of primary to secondary, tidal enhancement efficiency and helium abundance, would influence the binary evolution, thus influence the formation of BHk stars in binaries. Therefore, we discussed the effects of these input parameters on our results in this section, and we also discussed our results with recent observations and other theoretical models.

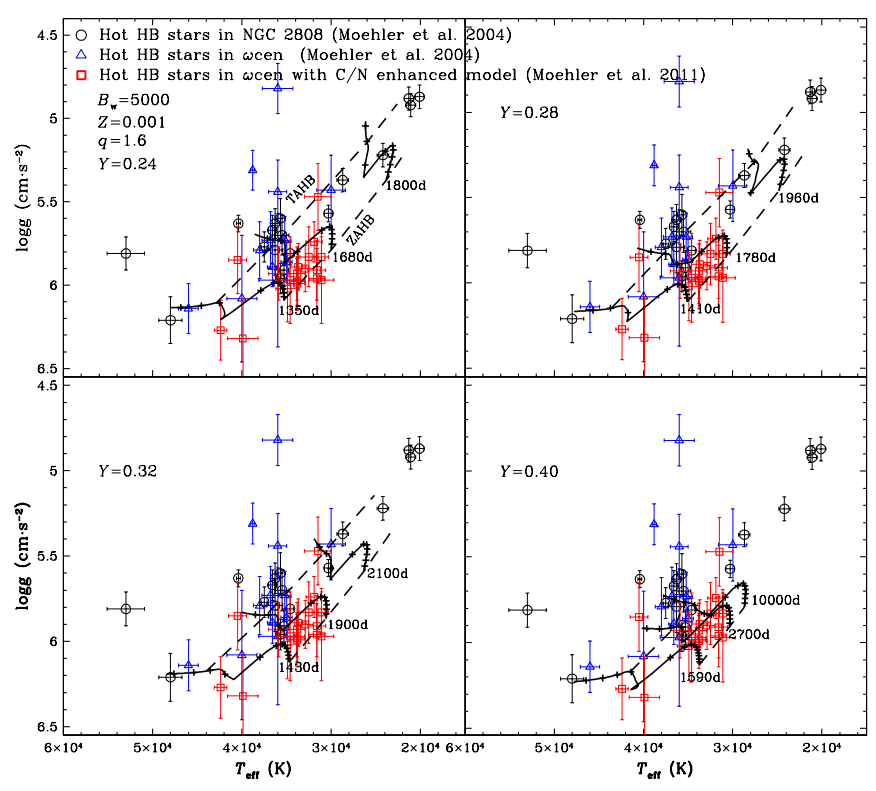

Figure 7. The same as Fig. 2, but for the comparison between observations and model VI.

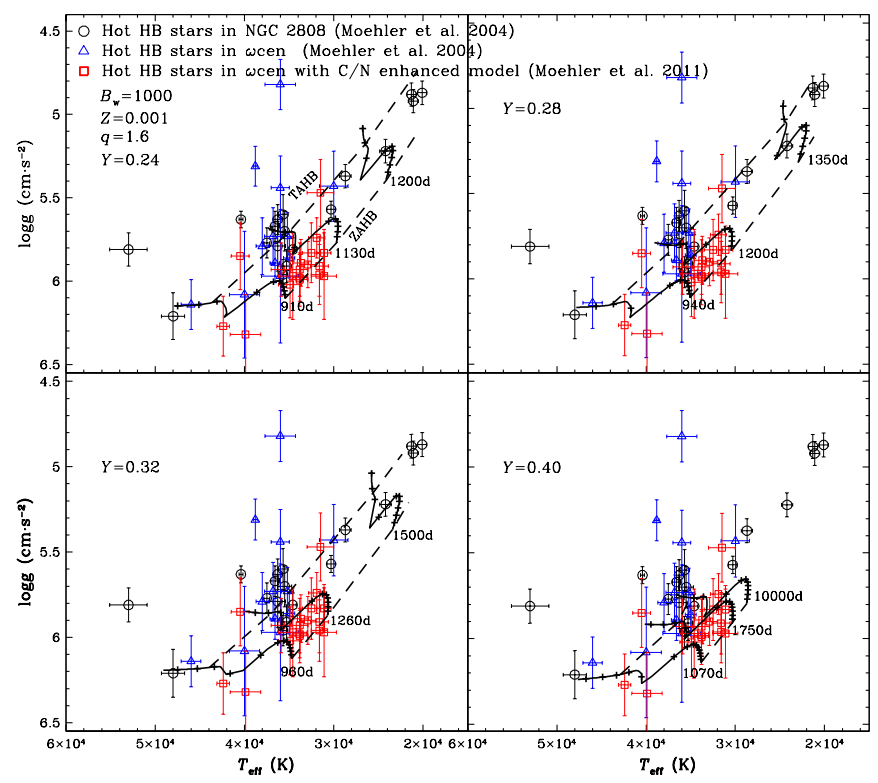

Figure 8. The same as Fig. 2, but for the comparison between observations and model VII.

\subsection{Metallicity, $Z$}

Fig 9 shows relationship between the range of initial orbital periods which is needed to produce BHk stars and the initial helium abundance for models I-III. These models have the same $q$ and $B_{\mathrm{w}}$ (i.e., $q=1.6, B_{\mathrm{w}}=10000$ ), but different metallicities (e.g., $Z=0.003,0.001$ and 0.0003 for models I, II and III respectively. ) The top dotted line in Fig. 9 represents the minimum orbital periods for the primary stars in model I to experience early hot flash (labeled by $P_{2}$ in Table 1), below which the primary stars would experience late hot flash and become BHk stars (it also can be considered as the maximum period to produced BHk stars, see Section 3.1). While the bottom dotted line denotes the minimum orbital periods for the primary stars in model I to experience late hot flash and become 


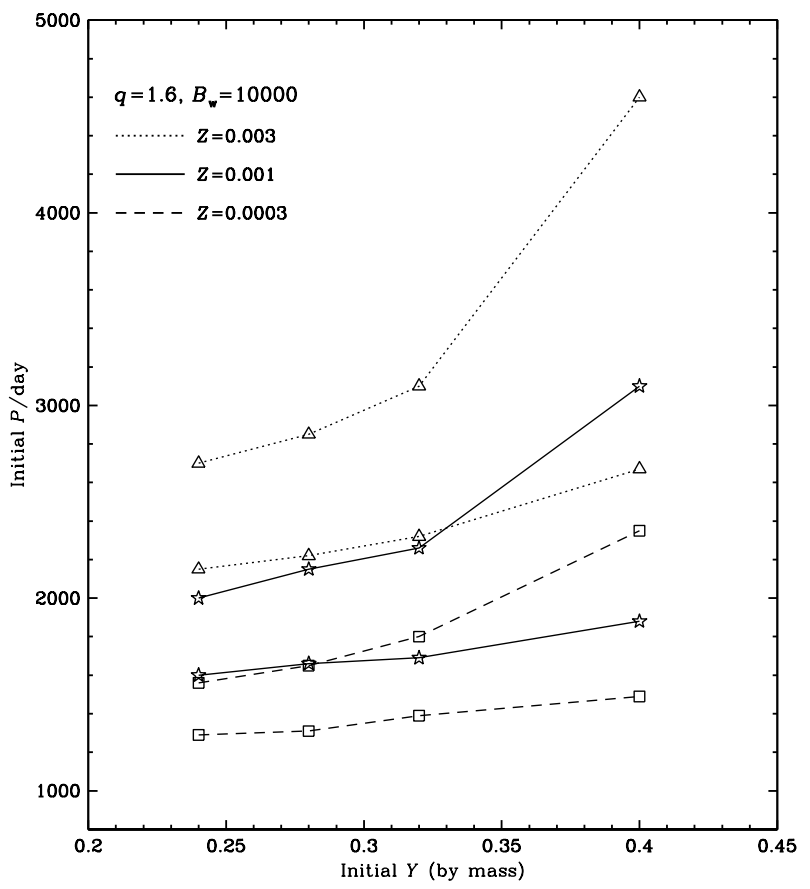

Figure 9. The correlation between the range of initial orbital periods to produce BHk stars with increasing helium abundance for different metallicities.

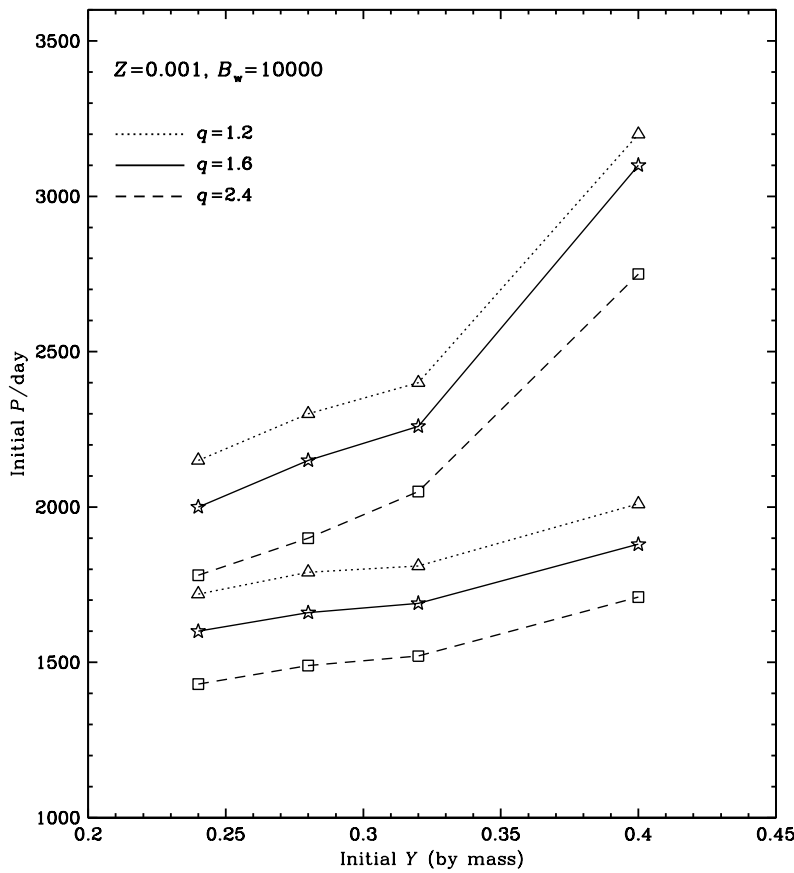

Figure 10. The correlation between the range of initial orbital periods to produce BHk stars with increasing helium abundance for different mass ratios.

BHk stars (labelled by $P_{3}$ in Table 1), below which the primary stars would lose too much envelope mass and die as helium WDs. Therefore, the region between the two dotted lines in Fig. 9 denotes the range of orbital periods in which the binaries would produce BHk stars for model I. Similarly, the region marked by two solid lines in Fig. 9 shows the range of orbital periods for the binaries in model II to produce BHk stars, while the region between two

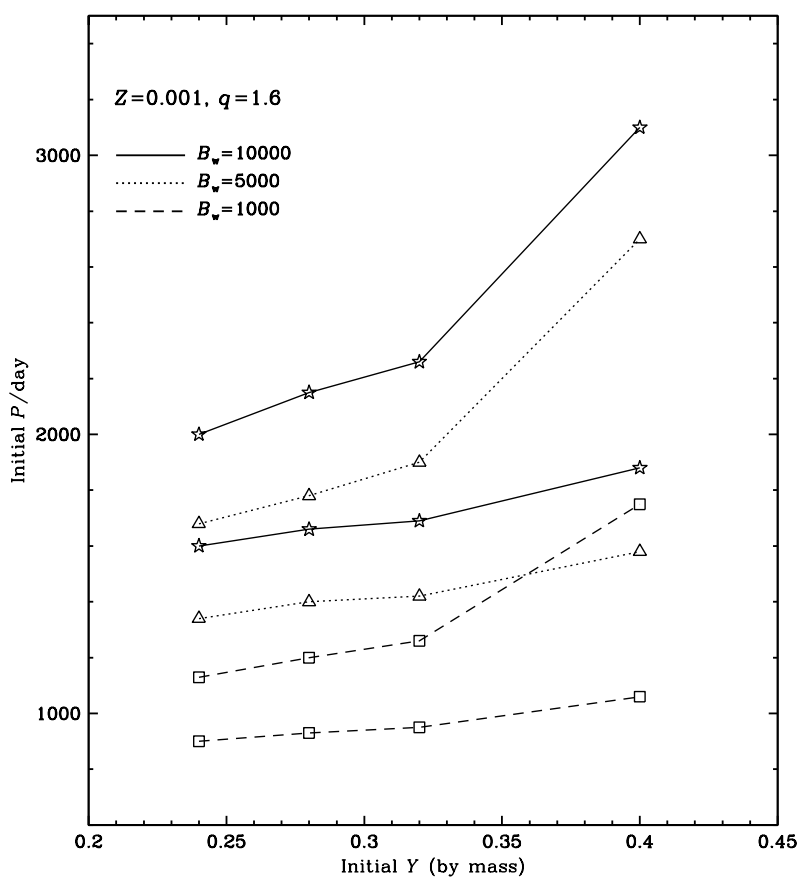

Figure 11. The correlation between the range of initial orbital periods to produce BHk stars with increasing helium abundance for different tidal enhancement efficiency.

dashed lines presents the range of orbital periods for the binaries in model III to produce BHk stars.

One can see from fig 9 that, with the metallicity increasing from model III to model I, the range of orbital period to produce BHk stars becomes wider. This trend is more evident at $Y=0.40$ for these three models, e.g., $P_{2}-P_{3} \approx 800 \mathrm{~d}$ for model III ( $Z=0.0003$ ) but increases to about $2000 \mathrm{~d}$ for model I $(Z=0.003)$. This is because that higher metallicity would lead to larger stellar wind on the RGB stage. Thus, a longer orbital period in binary also could lose enough envelope mass on the RGB stage to experience late hot flash and become BHk stars. It means that, in the tidally-enhancedstellar-wind scenario, GCs with higher metallicities would produce BHk stars more easily than metal-poor ones if a flat distribution of the orbital periods for binaries in GCs is expected. However, the detailed orbital periods for binaries and their distributions in GCs are difficult to obtained (Milone et al. 2012, 2016). Massive GC NGC 6388 and NGC 6441 are found to host extended HB morphologies and BHk stars (Rich et al. 1997; Busso et al. 2007) that would be hardly expected in the GCs with higher metallicity (e.g., $[\mathrm{Fe} / \mathrm{H}]=-$ 0.60 and -0.53 for NGC 6388 and NGC 6441 respectively; Harris 1996, version 2003). Therefore, our results obtained here would be a possible explanation for the formation of BHk stars in metalenriched GC NGC 6388 and NGC 6441. However, a further study for the effects of binaries on the formation of BHk stars in these two GCs is needed, including $N$-body simulation to calculate the distribution of orbital periods for the binaries in these two GCs. For model I in fig 9 (i.e., $Z=0.003$ ), with the initial helium abundance increasing, the orbital period range to produce BHk stars becomes larger (e.g., $P_{2}-P_{3} \approx 500 \mathrm{~d}$ for $Y=0.24$, but this value is about $2000 \mathrm{~d}$ for $Y=0.40$ ). This trend is also presented in models II and III in Fig. 9 that have initial metallicity of $Z=0.001$ and 0.0003 , respectively. We will discuss this result in Section 4.4. 


\subsection{Mass ratio, $q$}

To study the effects of mass ratio on our results, we showed in Fig 10 the relationship between the range of orbital periods that is needed to produced BHk stars and initial helium abundance for models II, IV and V listed in Table 1. These three models have the same metallicity $(Z=0.001)$ and tidal enhancement efficiency $\left(B_{\mathrm{w}}=10000\right)$, but with different mass ratios (e.g., $q=1.6,1.2$ and 2.4 for models II, IV and V, respectively). As we described in Fig. 9 , the region defined by the two lines with same type denotes the range of orbital periods in which BHk stars could be produced in binaries. Specifically, the top lines in each pair represents the maximum orbital periods to produce BHk stars, while the bottom lines in each pair represents the minimum orbital periods to produce $\mathrm{BHk}$ stars. From top to bottom of fig 10, the region defined by dotted lines is the range of orbital periods to produce BHk star for binaries in model IV $(q=1.2)$, and the region defined by solid lines are is the orbital periods range to produce BHk stars for the binaries in model II $(q=1.6)$, while the two dashed lines define the orbital periods range for the binaries in model $\mathrm{V}(q=2.4)$ to produce BHk stars.

One can see from Fig. 10 that the ranges of orbital periods to produce BHk stars are very similar from $Y=0.24$ to $Y=0.40$ among the three models with different mass ratios. It means that no evident correlations between mass ratio and the range of orbital periods that is needed to produce BHk stars in binaries are found. This is because of the fact that, in tidally enhanced stellar-wind model, smaller mass ratio (or more massive secondary) would expect much mass-loss for the primary, thus the orbital periods needed to produce BHk stars would be longer than the values for binaries with larger mass ratio. However, the whole space range of orbital periods to produce BHk star is not altered obviously. On the other hand, the space range of orbital periods to produce BHk stars for each model becomes wider with the increase of initial helium abundance, which is also found in Fig 9.

\subsection{Tidal enhancement efficiency, $B_{\mathrm{w}}$}

The tidal enhancement efficiency, $B_{\mathrm{w}}$, is a free parameter in tidallyenhanced-stellar-wind model. Tout \& Eggleton (1988) used a value of 10000 to explain the mass inversion phenomenon found in some RS CVn type binaries (Popper \& Ulrich 1977; Popper 1980). We adopted three values of $B_{\mathrm{w}}$ in our calculation to study its effects on our final results. Fig. 11 shows the relationship between the space range of orbital periods to produce BHk stars and the initial helium abundance for the binaries in models II, VI and VII. These three models have the same metallicity $(Z=0.001)$ and mass ratio $(q=1.6)$, but with different $B_{\mathrm{w}}$ (e.g., $B_{\mathrm{w}}=10000,5000$ and 1000 for models II, VI and VII, respectively; see Table 1). The lines in pair with different types have the same meaning as we described in figs 9 and 10. The two solid lines mark the space range of orbital periods which is needed to produce BHk stars for the binaries in model II $\left(B_{\mathrm{w}}=10000\right)$; the two dotted lines define the space range of orbital periods to produce BHk stars for the binaries in model VI $\left(B_{\mathrm{w}}=5000\right)$, while the space range marked by two dashed lines denotes the orbital periods to produce BHk stars for model VII $\left(B_{\mathrm{w}}=1000\right)$.

As expected, for a smaller value of $B_{\mathrm{w}}$, the orbital periods needed for binaries to produce BHk stars are shorter than the ones needed for the binaries with larger value of $B_{\mathrm{w}}$ to produce BHk stars. When the value of tidal enhancement increasing from 1000 to 10000 in fig 11 , the space range of orbital periods for the bi- naries to produce BHk stars would become a little larger (e.g., for $Y=0.40, P_{2}-P_{3} \approx 700,1100$ and $1200 \mathrm{~d}$ for $B_{\mathrm{w}}=1000,5000$ and 10000 respectively), but this trend is not very evident at lower $Y$, as we discussed in Paper I. We also found in fig. 11 that with the initial helium abundance increasing in each model, the space range of orbital periods for binaries to produce BHk stars becomes wider, especially for the highest helium abundance, $Y=0.40$, which is the same as we see in fig 9 and 10 .

\subsection{Helium abundance, $Y$}

For each model showed in figs 9-11, the space range of orbital periods for binaries to produce BHk stars becomes wider obviously with the increase in initial helium abundance. This trend is very evident for all the models regardless of the adopted metallicities, mass ratios and tidal enhancement efficiency. This is due to the fact that stars with higher helium abundance evolve faster than normal stars. For a fixed age at the RGB tip, helium enriched stars have smaller stellar masses at ZAMS ( $M_{\text {ZAMS }}$ ) than normal stars. Therefore, even in a wider binary system, these stars still could experience late hot helium flash and become BHk stars after losing a little envelope mass on the RGB stage, and the range of initial orbital periods needed to produce BHk stars in binaries with higher helium abundance is wider than the ones in binaries with lower helium abundance. Our result presented here means that helium abundance would be an important parameters in our binary models. With a higher value of $Y$, one would expect that BHk stars could be produced more easily in binaries if a flat distribution of the orbital periods is assumed in GCs. However, the detailed distribution of orbital periods for binaries in GCs is difficult to obtain from observation, but $\mathrm{N}$-body simulation may be an alternative option.

Recently, multiple populations are found as a common phenomenon among Galactic GCs, e.g., the splitting of MS, light elements anomalies (Piotto et al. 2007; Gratton Carratta \& Bragaglia 2012; Gratton et al. 2013, 2014). Helium enhancement due to selfenrichment in GCs is considered to be correlated with this phenomenon (D'Antona et al. 2002, D'Antona \& Caloi 2008; Milone 2015). When these heium enhanced second-generation stars evolve into HB stage, they would occupy BHB even EHB positions due to their higher helium abundance, and some of them would become BHk stars (D'Antona et al. 2002; 2010). These results seem to be consistent with the results we presented here such that BHk stars would be produced more easily in binary systems with higher initial helium abundance.

\subsection{Comparison with other models}

D'Antona et al. (2010) proposed that the BHk stars would be the progeny of the blue MS in $\omega$ Cen, which could be explained by using enhanced helium abundance of $Y=0.38-0.40$ (Moehler et al. 2007). They assumed that these blue MS stars may experience extra mixing at upper RGB, which could increase the surface helium abundance up to $Y \approx 0.8$. Then these stars evolve along a noncanonical evolution tracks and settle on BHk regions when central helium ignites. However, the helium enhanced model proposed by D'Antona et al. (2010) could not predict other chemical anomalies in the stellar surface, such as carbon and nitrogen. In fact, using the MXU mode of the Focal Reducer and Spectrograph of the Very Large Telescope, Latour et al. (2014) found a clear positive relationship between helium and carbon enhancement in some $\mathrm{BHk}$ stars of $\omega$ Cen, which is also predicted by our model (see carbon 
and helium abundance of 'late' models listed in Table 2, also see the discussion in paper I. Note that we do not consider chemical diffusion and gravitational settling process in our models). This observation evidence favors late hot flash scenario for the formation of BHk stars in GCs.

Nevertheless, the luminosity (or magnitude) extension of BHk stars in H-R diagram (or CMD) of some GCs is difficult to understand for most of the scenarios explaining the formation of $\mathrm{BHk}$ stars (Brown et al. 2010; D'Antona et al. 2010; Paper I), including late hot flash scenario. Recently, Tailo et al (2015) suggested that a rapidly rotating second-generation progenitor for BHk stars in $\omega$ Cen would result in a larger helium core mass by up to $\sim 0.04 M_{\odot}$ when helium core flash is taking place, thus a higher luminosity for stars at ZAHB. They suggested that the pre-MS accretion disc of progenitors for BHk stars may suffer an early disruption, which results in a faster rotation for the following evolution and thus an increase in helium core mass at helium flash. Employing this mechanism, they could explain the luminosity range of BHk stars in $\omega$ Cen. Note that the results in Tailo et al (2015) would not conflict with our results obtained in this study. In Tailo et al (2015), the BHk progenitors also need to experience huge mass loss on the RGB stage and undergo late hot helium flash before becoming BHk stars. However, they did not consider the physical mechanism of huge mass loss on the RGB in details. On the other hand, in our models, tidally enhanced stellar wind in binary evolution naturally provides a physical mechanism for mass loss on the RGB stage in late hot flash scenario, but we did not consider the effects of stellar rotation on our final results, and it will be studied in the near future.

\subsection{The role of binaries on formation of BHk stars in GCs}

As we can see from this study, the orbital periods needed to produce BHk stars due to the tidally enhanced stellar wind in binaries are relative long (e.g., from about 900 to $4600 \mathrm{~d}$ depends on the models list in Table 1) when comparing with close binaries, thus these binaries would not be found easily in GCs from observation due to their long orbital periods. Moreover, due to the dense environment in GCs, soft binaries (i.e., binaries with longer orbital periods) woud be destroyed easily during the dynamical evolution of GCs (Hut et al. 1992; Hurley, Aarseth \& Shara 2007; Heber 2016). The boundary between hard and soft binaries depends on the mean kinetic energy of the cluster stars (Hut et al. 1992). A useful method was adopted by Hurley, Aarseth \& Shara (2007) to estimate the boundary between hard and soft binaries in terms of binary separation, which is given by twice the cluster half-mass radius divided by $N$. In this method, $N$ is the total number of cluster members, including single and binary stars. We used this method to estimate the boundary of GC $\omega$ Cen as well, which was compared with our theoretical results in this study. From the catalogue compiled by Harris (1996, version 2003), the half-mass radius of $\omega$ Cen is about 4.18 arcmin; considering that its distance from the sun is about $5.3 \mathrm{kpc}$, we could obtain the half-mass radius for this GC is to be $6.44 \mathrm{pc}$. By assuming $N=10^{6}$ in this GC, we estimate the boundary between hard and soft binaries in terms of separation is about 2.66 $\mathrm{au}$ (Here au is the abbreviation of 'astronomical unit'). This separation corresponds to an orbital period of about $1400 \mathrm{~d}$ for a binary where the masses of primary and secondary are 0.83 and $0.52 M_{\odot}$, respectively. If this is the case, at least part of binaries in our model which produce BHk stars could be classified as hard binaries and would survive during the evolution of GC. However, we also estimated a hard/soft boundary of about 1.46 au in GC NGC 2808 using the same method and it corresponds to an orbital period of about $500 \mathrm{~d}$ for a binary system with the mass of two components are 0.83 and $0.52 M_{\odot}$, respectively. This boundary is much shorter than the orbital periods in our models needed to produce BHk stars. Note that, the orbital periods needed to produce BHk stars in our models become shorter with a smaller tidal enhancement efficiency (i.e., $B_{\mathrm{w}}$ ) or a larger ratio of primary to secondary (see figs 10 and 11 in this study). Therefore, this discrepancy could be resolved by using a smaller $B_{\mathrm{w}}$ and a larger ratio of primary to secondary in our models.

Castellani et al. (2006) found a peculiar group of stars, which are called HBp stars in their study, near the HB of GC NGC 2808 using the WFPC2 camera on board the Hubble Space Telescope. These peculiar stars locate in the region of blue straggler stars in optical CMD of NGC 2808, but very close to the hot HB stars in ultraviolet CMD (see fig 2 in their study). They proposed that this phenomena is possibly caused by the composite nature of the spectrum which probably results from the photometric blends of physical binarity (e.g, the faint companion stars have not been detected; see Allard et al. 1994). By assuming the blended star (the faint companion star in a binary) as an MS star, Castellani et al. (2006) found that a hot HB star could be shifted to the HBp region in the CMD due to a blend with a redder companion, and the faintest and hottest HB stars presented the maximum shift. Their results indicated that at least some of the blue and hottest HB stars in NGC 2808 are binaries.

Due to the long orbital periods, the binaries consisting of BHk stars predicted by our models are difficult to be found directly in GCs from the observation, but there may be some indirect evidences to test our scenario. Following the results we presented here, if the initial orbital periods of the binaries shorter than the minimum values needed to produce BHk stars in GCs (e.g., the orbital periods labelled by $P_{3}$ in Table 1), the primary stars in these binaries would fail to ignite helium burning in their cores and die as helium WDs. On the other hand, if the initial orbital periods are short enough, the primary star in a binary would fill its Roche lobe radius and transfer mass to the secondary through Roche lobe overflow (RLOF). This kind of mechanism also would form hot EHB stars (see Han et al. 2002, 2003). However, when tidally enhanced stellar wind is considered into binary evolution, the initial orbital period which is required for the occurrence of RLOF in binaries would become much shorter. In the study of Tout \& Eggleton (1988), when tidally enhanced stellar wind is not considered in binary evolution, the initial orbital periods required for RLOF in binaries are nearly shorter than $1500 \mathrm{~d}$ for a primary star with a mass of $2 M_{\odot}$, depending on the mass ratio adopted. However, the required initial orbital periods for RLOF decrease to less than $30 \mathrm{~d}$ if tidally enhanced stellar wind is considered (see figs 1 and 2 in their study), and the final orbital periods (e.g., at the point when RLOF begins in a binary) for these binaries are nearly less than $100 \mathrm{~d}$ (see fig 3 in Tout \& Eggleton 1988). For a less massive primary star (i.e., $1.3 M_{\odot}$ ), these initial orbital periods required for RLOF become even more shorter, e.g., less than $10 \mathrm{~d}$ (see fig 4 in Tout \& Eggleton 1988). Therefore, based on these results and the results we presented in this study, helium WD binaries would be formed through tidally enhanced stellar wind in our models if the initial orbital periods are in the range of several tens to hundreds of days. Thus these kind of binaries present much shorter orbital periods than the binaries consisting of BHk stars predicted by our models, and they would be detected more easily from the observations. If these kinds of binaries are found in GCs, it is an indirect evidence to support our scenario presented here.

Actually, Kaluzny et al. (2007, 2013, 2014, 2015) have done 
a series of study on detached eclipsing binaries (DEBs) in GCs. They aimed to obtain the masses, orbital periods, radius and luminosity of these binary components with higher precision, finally to determine the ages and distances of the GCs which could be used to test the stellar evolution models (Kaluzy et al. 2005). Among the DEBs listed in these literatures, most of the binaries present short orbital periods (e.g., several days to more than $10 \mathrm{~d}$ ), and the two components of these binaries are usually MS stars with nearly equal masses. However, Kaluzny et al. (2013) found a DEB named V69 in GC M4, which presents an orbital period of $48.19 \mathrm{~d}$. The two components are MS stars near the turn-off point in the CMD of M4, with a mass of $0.7665 \pm 0.0053 M_{\odot}$ for the primary and a mass of $0.7278 \pm 0.0048 M_{\odot}$ for the secondary. This binary is a possible candidate system to form the helium WD binaries through tidally enhanced stellar wind. Following our scenario, the primary star in this binary system would be probably lose huge envelope mass at the RGB stage through tidally enhanced stellar wind rather than through RLOF, and become a helium WD in a binary system with a orbital period of several ten days to more than $100 \mathrm{~d}$.

\section{CONCLUSIONS}

As a further study for Paper I, by considering tidally enhanced stellar wind into binary evolution, we studied in detail the effects of metallicity, mass ratio, tidal enhancement efficiency and helium abundance on the formation of BHk stars . Over 20 sets of binary models combined with different values of parameters mentioned above are adopted to study their effects on the formation of BHk stars. For each set of models, the space range of initial orbital periods which are needed to produce BHk stars for binaries was presented. We also showed the evolution tracks of primary stars from ZAMS to WD including the stage of late hot helium flash, as well as the evolution parameters on the ZAHB for some primary stars.

Our results are compared with the observation in $T_{\text {eff }}-\log g$ plane. Even though the input parameters of these binary models are different from each other, all binaries in these models could produce BHk stars within different range of initial orbital periods. Most of the BHk stars in NGC 2808 and $\omega$ Cen locate well in the region predicted by our models, especially when $\mathrm{C} / \mathrm{N}$-enhanced model atmospheres are considered in obtaining the stellar atmosphere parameters of BHk stars. The effects of metallicity, mass ratio, tidal enhancement efficiency and helium abundance on the space range of initial orbital periods needed to produce BHk stars are discussed in detail. We found that mass ratio of primary to secondary and tidal enhancement efficiency have little effects on the formation of BHk stars in our model, while metallicity and helium abundance seem to play important roles in formation of BHk stars in binaries. Especially, with the helium abundance increasing from $Y=0.24-0.40$, the range of initial orbital period to produce BHk stars becomes wider obviously for all the models. Assuming a flat initial orbital periods distribution for binaries in GCs, one would expect a much easier production of BHk stars if these stars have higher initial helium abundance. Our results presented here indicate that tidally enhanced stellar wind in binary evolution is a possible formation channel for BHk stars in GCs, but further studies and more evidences are needed to investigate the roles of binaries on this problem before it comes to a conclusive result.

\section{ACKNOWLEDGEMENTS}

We thank the anonymous referee for valuable comments and suggestions that helped us to improve the paper. This work is supported by the National Natural Science Foundation of China (Grant Nos, 11503016, 11390371, 11233004, 11573061), the Youth Fund project of Hunan Provincial Education Department (Grant No. 15B214). ZH is partly supported by the Natural Science Foundation of China (Grant No. 11390374, 11521303), the Science and Technology Innovation Talent Programme of the Yunnan Province (Grant No. 2013HA005) and the Chinese Academy of Sciences (Grant No. XDB09010202, KJZD-EW-M06-01).

\section{REFERENCES}

Allard, F., Wesemael, F., Fontaine, G., Bergeron, P., \& Lamontagne, R., 1994, AJ, 107, 1565

Bedin, L. R., Piotto, G., Anderson, J., Cassisi, S., King I. R., Momany Y., Carraro G., 2004, ApJ, 605, L125

Bellazzini, M. et al., 2008, AJ, 136, 1147

Brown, T. M., Lanz, T., Sweigart, A. V. et al., 2012, ApJ, 748, 85

Brown, T. M., Sweigart, A. V., Lanz, T. et al., 2001, ApJ, 562, 368

Brown, T. M., Sweigart, A. V., Lanz, T. et al., 2010, ApJ, 718, 1332

Busso, G., Cassisi, S., Piotto, G., Castellani, M. et al., 2007, A\&A, 474, 105

Cassisi, S., Schlattl, H., Salaris, M., \& Weiss, A., 2003, ApJ, 582, L43

Castellani, M., \& Castellani, V., 1993, ApJ, 407, 649

Castellani, V., Iannicola, G., Bono, G. et al., 2006, A\&A, 446, 569

Catelan, M., 2009, Ap\&SS, 320, 261

D’Antona, F., \& Caloi, V., 2008, MNRAS, 390, 693

D'Antona, F., Caloi, V., \& Ventura, P., 2010, MNRAS, 405, 2295

D’Antona, F., Caloi, V., Montalbán, J., Ventura, P., \& Gratton, R., 2002, A\&A, 395, 69

D’Cruz, N. L., Dorman, B., Rood, R. T. et al., 2000, ApJ, 530, 352

D'Cruz, N. L., Dorman, B., Rood, R. T., \& O'Connell, R. W., 1996, ApJ, 466, 359

Dinescu, D. I., Girard, T. M., \& van Altena, W. F., 1999, AJ, 117, 1792

Dupree, A. K., Smith, G. H., Strader, J., et al., 2009, AJ, 138, 1485.

Gratton, R. G., Carretta, E., Bragaglia, A. et al., 2010, A\&A, 517, A81

Gratton, R. G., Carretta, E., \& Bragaglia, A., 2012, A\&ARv, 20, 50

Gratton, R. G., Lucatello, S; Sollima, A. et al., 2013 A\&A, 549, A41

Gratton, R. G., Lucatello, S; Sollima, A. et al., 2014 A\&A, 563, A13

Han Z. W., Lei Z. X., 2014, in van Grootel V., Green E., Fontaine G., Charpinet S. (eds), ASP Conf Ser. Vol. 481, Sixth Meeting on Hot Subdwarf Stars and Related Objects. Astron. Soc. Pac., San Francisco, p. 213

Han Z., Chen X., Lei Z., 2010, in Kologera V., van der Sluys M. (eds), Proc. AIP Conf. Vol. 1314, International Conference on Binaries: In Celebration of Ron Webbinks 65th Birthday. American Inst. Phys., New York, p. 85

Han Z., Chen X., Lei Z., Podsiadlowski P., 2012, in Kilkenny D., Jeffery C. S., Koen C., eds, ASP Conf. Ser. Vol. 452, Fifth Meet- 
ing on Hot Subdwarf Stars and Related Objects. Astron. Soc. Pac., San Francisco, p. 3

Han, Z., Podsiadlowski, Ph., Maxted, P. F. L., \& Marsh, T. R., 2003, MNRAS, 341, 669

Han, Z., Podsiadlowski, Ph., Maxted, P. F. L., Marsh, T. R., \& Ivanova, N., 2002, MNRAS, 336, 449

Harris, W. E. 1996, AJ, 112, 1487

Heber, U., 2016, PASP, 128, 2001

Hoyle, F., \& Schwarzschild, M., 1955, ApJS, 2, 1

Hurley J. R., Aarseth S. J., Shara M. M., 2007, ApJ, 665, 707

Hut, P., McMillan, S., Goodman, J., et al., 1992, PASP, 104, 981

Jiang, D. K., Han, Z. W., \& Li, L. F., ApJ, 2014, 789, 88

Johnson, C. I., \& Pilachowski, C. A., 2010, ApJ, 722, 1373

Kaluzny, J., Thompson, I. B., Dotter, A., et al., 2015, AJ, 150, 155

Kaluzny, J., Thompson, I. B., Dotter, A., et al., 2014, AcA, 64, 11

Kaluzny, J., Thompson, I. B., Rozyczka, M., et al., 2013, AJ, 145, 43

Kaluzny, J., Rucinski, S. M., Thompson, I. B., Pych, W., \& Krzeminski, W., 2007, AJ, 133, 2457

Kaluzny, J., Thompson, I. B., Krzeminski, W., et al. 2005, in AIP Conf. Proc. Vol. 752, Stellar Astrophysics with the World's Largest Telescopes, Am. Inst. Phys., New York, p.70

Lanz, T., Brown, T., Swegart, A., HUbeny, I., \& Landsman, W., 2004, ApJ, 602, 342

Latour, M., Randall, S. K., Fontaine, G., Bono, G., Calamida, A., \& Brassard, P., 2014, ApJ, 795, 106

Lee, Y.-W., 2005, ApJ, 621, L60

Lei, Z. X., Chen, X. F., Zhang, F. H., \& Han, Z. W., 2013a, A\&A, 549, A145

Lei, Z. X., Zhang, F. H., Ge, H. W., \& Han, Z. W., 2013b, A\&A, 554, A130

Lei, Z. X., Chen, X. M., Zhang, F. H., \& Han, Z. W., 2015, MNRAS, 449, 2741 (Paper I)

McDonald, I., \& Zijlstra, A. A., 2015, MNRAS, 448, 502

Marconi, M., Musella, I., Criscienzo, M. D. et al., 2014, ASPC, 486, 203

Marino, A. F., Milone, A. P., Przybilla, N. et al., 2014, MNRAS, 437, 1609

Miller Bertolami, M. M., Althaus L. G., Unglaub, K., \& Weiss, A., 2008, A\&A, 253, 265

Milone, A. P., 2015, MNRAS, 446, 1672

Milone, A. P., Marino, A. F., Bedin, L. R., Dotter, A. et al., 2016, MNRAS, 455, 3009

Milone, A. P., Piotto, G., Bedin, L. R., Aparicio, A. et al., 2012, A\&A, 540, 16

Moehler, S., Dreizler, S., Lanz, T. et al., 2007, A\&A, 575, L5

Moehler, S., Dreizler, S., Lanz, T. et al., 2011, A\&A, 526, A136

Moehler, S., Sweigart, A. V., Landsman, W. B. et al., 2004, A\&A, 415, 313

Moni Bidin, C., Villanova, S., Piotto, G. et al., 2012, A\&A, 547, A109

Paxton, B., Bildsten, L., Dotter, A. et al., 2011, ApJS, 192, 3

Paxton, B., Cantiello, M., Arras, P. et al., 2013, ApJS, 208, 4

Paxton, B., Marchant, P., Schwab, J. et al., 2015, ApJS, 220, 15

Piotto, G., Bedin, L. R., Anderson, J. et al., 2007, ApJ, 661, L53

Piotto, G., King, I. R., Djorgovski, S. G. et al., 2002, A\&A, 391, 945

Piotto, G., Villanova, S., Bedin, L., Gratton, R. et al., 2005, ApJ, 621,777

Popper D. M. 1980, ARA\&A, 18, 115

Popper D. M., \& Ulrich R. K. 1977, ApJ, 212, L131

Reimers, D., 1975, MSRSL, 8, 369
Renzini, A., \& Fusi Pecci, F. 1988, ARA\&A, 26, 245

Rich, R. M., Sosin, Craig., Djorgovski, S. et al., 1997, ApJ, 484, 25

Tailo, M., D’Antona, F., Vesperini, E., et al., 2015, Natur, 523, 318

Tout, C. A., \& Eggleton, P. P., 1988, MNRAS, 231, 823

Villanova, S., Geisler, D., Gratton, R. G., Cassisi, S., 2014, ApJ, 791, 107

Villanova, S., Piotto, G., King, I. R. et al., 2007, ApJ, 663, 296

Whitney, J. H., Rood, R. T., O'Connell, R. W. et al., 1998, ApJ, 495, 284

Willson, L. A., 2000, ARA\&A, 38, 573 